\title{
Pharmacognostic Evaluation of Curcumin on Diabetic Retinopathy in Alloxan-induced Diabetes through NF-KB and Brn3a Related Mechanism
}

\author{
Debasish Pradhan ${ }^{1 *}$, Toffa Dasmohapatra ${ }^{2}$, Gitanjali Tripathy ${ }^{3}$
}

\section{Debasish Pradhan ${ }^{1 *}$, Toffa Dasmohapatra², Gitanjali Tripathy ${ }^{3}$}

\author{
'Department of Pharmaceutical \\ Sciences, Creighton University, Carlifor- \\ nia Plaza,Omaha,NE68102, USA. \\ ${ }^{2}$ University Department of \\ Pharmaceutical Sciences, Utkal \\ University,Odisha,INDIA. \\ ${ }^{3}$ Department Of Pharmacy, Govern- \\ ment Polytechnic, Chandrashekharpur, \\ Odisha,INDIA.
}

\section{Correspondence}

Dr. Debasish Pradhan

Department of Pharmaceutical Sciences, Creighton University, 2500 Carlifornia Plaza, Omaha, NE68102, USA.

Phone No: 9861137772

E mail Id: deba_udps@yahoo.co.in

History

- Submission Date: 26-10-2017;

- Review completed: 19-12-2017;

- Accepted Date: 10-01-2018

DOI : 10.5530/pj.2018.2.56

Article Available online

http://www.phcogj.com/v10/i2

Copyright

(C) 2018 Phcog.Net. This is an openaccess article distributed under the terms of the Creative Commons Attribution 4.0 International license.

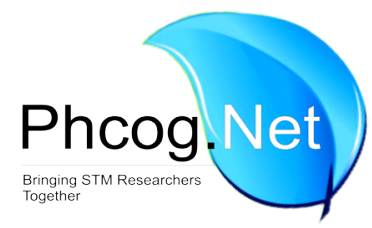

\begin{abstract}
Background: Diabetic retinopathy is one of the most common micro vascular complication of diabetes and involves an abnormal pathology of major retinal pigment epithelium, inter retinal oedema and intraocular neovascularisation where pro-inflammatory proteins including ICAM-1, iNOS and VEGF release by activation of enzyme CaMKII/NF-kB expression Diabetic induced oxidative stress followed by deactivation of Brn3a expression in the retinal ganglionic cells are also early events in pathogenesis of Diabetic retinopathy. These factors are important contributors to the development of clinically significant diabetic retinopathy. Objective: Objective of this study to examine the effect of curcumin with antioxidant and anti-inflammatory properties obtained from Curcuma longa against diabetes-induced retinal vascular damage and its mechanism of action by in-vivo in retinas of rat rendered diabetic by alloxan and in vitro in western blotting and RGC tissue culture. Method: We administered curcumin or saline vehicle to experimental animals daily for 12 weeks. Vascular permeability, expression of CaMK II/NF-kB, Retinal morphology and neuropathic change of the retinal ganglion cells were investigated. Results: As an anti-oxidant, curcumin raised Retinal Ganglionic cells by increasing Brn3a expression during oxidative stress condition and subsequently decreased the expression of inflammatory mediators such as VEGF, iNOS and ICAM-1 as an anti-inflammatory agent by inhibiting CaMKII and NF-kB expression. Conclusion: Curcumin, a common food additive has beneficial effects in experimental studies of diseases that are characterised by increased oxidative stress and inflammatory reactions. It appears to be a useful adjunct therapy to possibly inhibit the progression of retinopathy, sight threatening complication faced by diabetic patients.

Key words: Curcumin, Inflammation, Oxidative stress, CaKMII, NF-KB, Brn3a.
\end{abstract}

\section{INTRODUCTION}

Diabetic retinopathy, also known as diabetic eye disease, is when damage occurs to the retina due to diabetes. It can eventually lead to blindness. It affects up to 80 percent of people who have had diabetes for 20 years or more. At least $90 \%$ of new cases could be reduced if there were proper treatment and monitoring of the eyes. The longer a person has diabetes, the higher his or her chances of developing diabetic retinopathy. It is also the leading cause of blindness for people aged 20 to 64 years. ${ }^{1,2,10}$ Microvascular lesions ${ }^{22}$ such as microaneurysms, increased vascular permeability caused by the breakdown of the blood-retinal barrier (BRB), and capillary dropout are thought to be key causes of diabetic retinopathy. ${ }^{35,37}$ The sole purpose of retinal circulation is to support the metabolic demands of the inner retinal neurons and glial; these cells may also be damaged by the diabetic state. Retinal ganglion cells (RGC) are the sole output neurons from the eyes, assuming the critical role of transmitting visual signals to the higher visual center at the brain cortex before signal processing.

Curcumin, a pharmacological operator which is utilized as a part of Asian conventional medication because of its mitigating and hostile to oxidization highlights. Curcumin, a characteristic item that has been comprehensively examined by specialists from both natural and compound purpose for view. Curcumin having very nearly a two centuries old consistent history, starting from 1815, when curcumin was initially confined from turmeric. ${ }^{21}$ In the wake of finding of its threatening to development affect curcumin has most cherished subject for all branches of science including natural, inorganic and physical science. It is a $\alpha, \beta$-unsaturated- $\beta$ diketone (Figure 2) and central bioactive section of Curcuma longa has a place with family Zingiberaceae (Figure 1) which gives grouped tumor counteractive action operator and against inflammatory properties including assurance from metabolic dis-

Cite this article: Dr Pradhan D, Dasmohapatra T, Tripathy G. Pharmacognostic Evaluation of Curcumin on Diabetic Retinopathy in Alloxan-induced Diabetes through NF-KB and Brn3a Related Mechanism. Pharmacog J. 2018;10(2):324-32. 


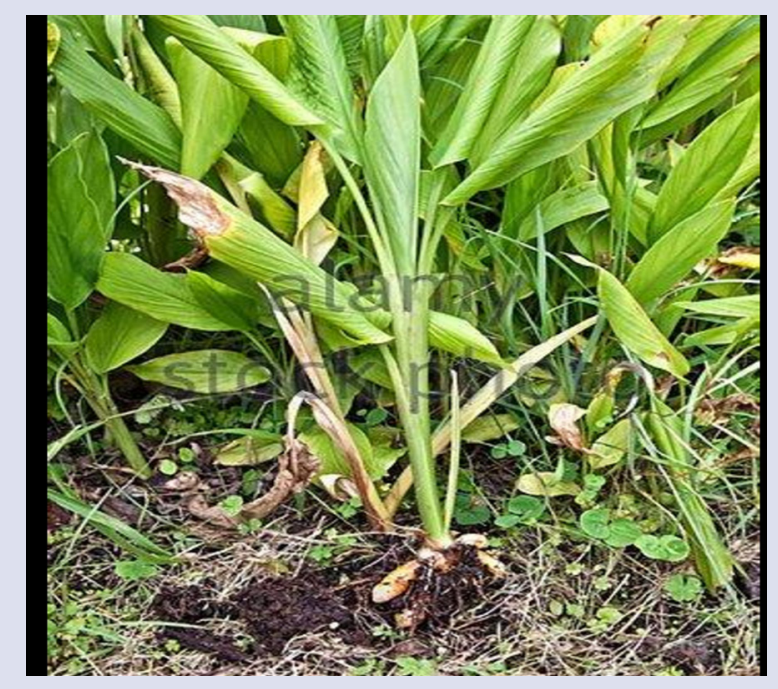

Figure 1: Image of Curcuma longa

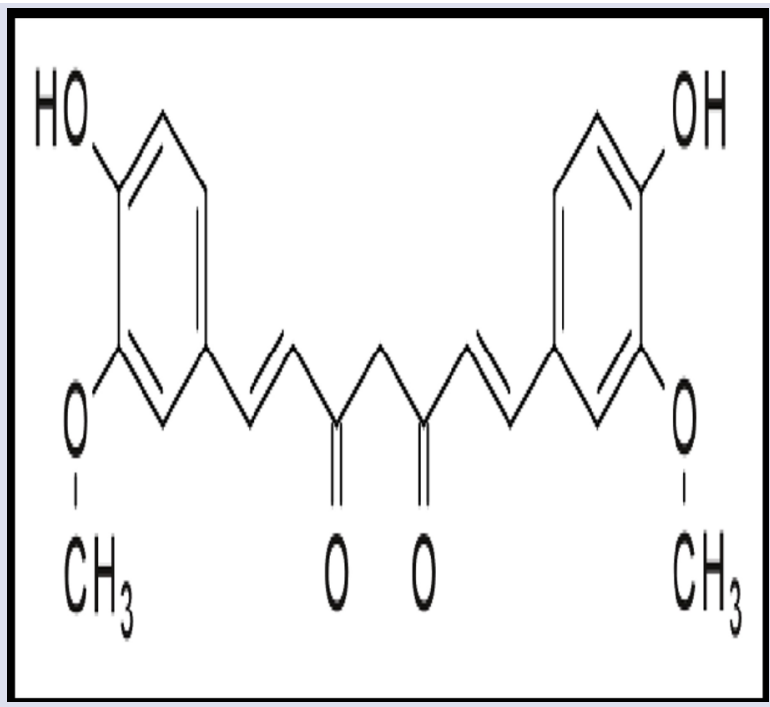

Figure 2: Chemical structure of Curcumin - 1E,6E)-1,7-Bis(4-hydroxy3-methoxyphenyl)-1,6 heptadiene-3,5-dione

orders. ${ }^{5}$ Hence it is used to treat diabetic retinopathy which hurts each and every genuine cell of retina, vascular cells and shading epithelial cells and makes driving purpose behind visual inadequacy around the world. Almost all patients with type I diabetes and more than $60 \%$ with type II diabetes have some level of retinopathy after 20 yrs. ${ }^{33,36}$ In the present review, we have researched the impact of curcumin on oxidative stress and inflammation in the retina of diabetes initiated by alloxan in rats for 12 weeks by comparing with control rats. The results of this research demonstrated that curcumin obtained from Curcuma longa reduces oxidant capacity of retina during oxidative stress condition inside the body by increasing Brn3a transcription factor expression and represses the level of inflammatory protein by diminishing activity of CaMKII and NF-kB transcription factor-intervened retinal vascular damage in patients with diabetic retinopathy. ${ }^{12,13}$

$\mathrm{Ca} 2+/$ calmodulin dependant protein kinase II (CaMKII) is a multifunctional serien/threonine protein kinase that coordinates destruction of neuronal cells including retinal ganglion cells and releases inflammatory mediators. Along these lines inhibitor of CaMKII and NF-kB transcription

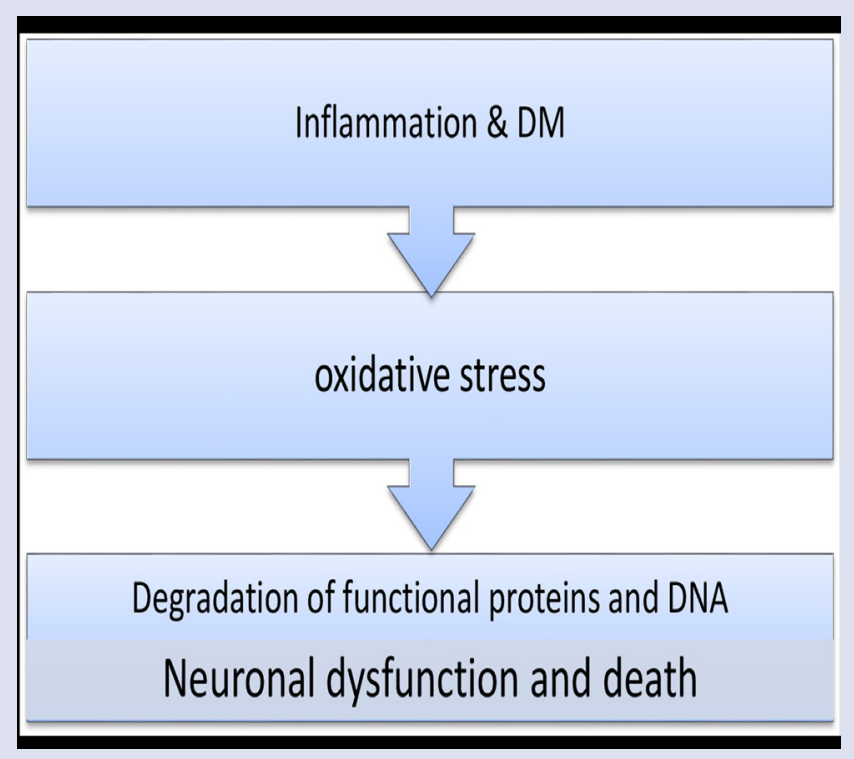

Figure 3: Diagram of Oxidative stress condition inside the body

factor, curcumin is strongly neuroprotective for neuron-crushing ailments, for instance, diabetic retinopathy. ${ }^{12,13,14,15}$

As shown (Figure 3) Oxidative stress shows up when there is a genuine irregularity between era of ROS and its clearance by cell antioxidant defences. Diabetic-prompts oxidative stress take after by enactment of Brn3a in the retina are early occasions in pathogenesis of diabetic retinopathy. Anti-oxidant properties of curcumin diminish era of ROS by activating of Brn3a factor in the retina. ${ }^{3,6,8,9}$

\section{MATERIALS}

Chemials were reagent-review quality and were obtained from Sigma Aldrich, Neheru Nagar, Mumbai.

\section{Extraction of Curcumin from Curcuma longa and Detection}

Depending on its origin and the soil conditions where it is grown, turmeric contains $2 \%-9 \%$ curcuminoids. The word "curcuminoid" indicates a group of compounds such as curcumin, demethoxycurcumin and bis-demethoxycurcumin and cyclic curcumin. Out of these, curcumin is the major component. Hence Solvent extraction followed by column chromatography has been followed by us for separating Curcumin from turmeric. Ethanol has been used as a most preferred solvent for extracting curcumin in temperature range of 60 to 80 -degree celcius. Curcumin was separated from curcumin mix (a mixture of Curcumin, demethoxycurcumin and bisdemethoxycurcumin) by column chromatography by adsorbing mixture on siliaca gel using solvent like ethanol to yield three different fractions, the curcumin fraction was further purified on silica gel using ethanol as eluent. For detection of curcumin in the UV region using a common detection of wavelength in range $260 \mathrm{~nm}$ was used. ${ }^{11,25}$

\section{Animals}

In this experiment study, male spargue -Dawley rats (8 weeks old) weighing $245 \mathrm{~g}$ were kept under a $12 \mathrm{~h}$ dark cycle, the room temperature was maintained in the range of 23 -degree celcius of $50 \%-70 \%$ humidity. The experiments were approved by the Institutional Animal Care and Use Subcommittee at our university. Food and water were available. Rats 
were fasted for $16 \mathrm{~h}$ and randomly divided into 2 groups. First groups of Rats was administered a $40 \mathrm{mg}$ dose of Alloxan/kg intraperitonially, while second group of rats was administered only citrate buffer $(0.1 \mathrm{~mol} / \mathrm{L}, \mathrm{pH}$ $4.5)$ according to references. ${ }^{28,29}$ Blood glucose levels were monitored $72 \mathrm{~h}$ later after Alloxan or vehicle injection, at regular intervals of every week throughout the study and immediately prior to euthanasia, blood samples were obtained by tail prick, and blood glucose concentration measured using a blood glucose meter. Only rats with fasting glucose concentrations ( $\geq 300 \mathrm{mg} / \mathrm{dL}$ ) were included in the DM group. The first group of rats became diabetic as their blood glucose level was more than $\geq 300 \mathrm{mg} / \mathrm{dl}$. Insulin was administered to diabetic rats to permit moderate weight pick up while keeping up hyperglycaemia. 3-4 days in the wake of actuating diabetes, rats were isolated into two groups Diabetic rats and Diabetic rats with curcumin at a dosage of $100 \mathrm{mg} / \mathrm{kg} /$ day. ${ }^{7}$ Each group had 3 rats and whole rat colony got crisp powdered eating routine weekly. The rats were measured two times each week and their nourishment utilization were measured once every week. A total 6 rats were developed into diabetes and been divided into 2 groups and fed with 50:50 mix of normal saline and dimethyl sulfoxide (DMSO, placebo, $N=6)$ or curcumin in $50 \%$ DMSO $(N=3)$ at $5 \mathrm{mg} / \mathrm{kg}$. Rats in the sham group received standard husbandry care, gavage feeded with PBS, but were not treated with Alloxan. At the end of 12 weeks rats were euthanized and retinas harvested for histological and molecular study. After 12 weeks diabetic rats were dark adapted for $1 \mathrm{hr}$ and they were anesthetized with an intraperitonial infusion of $1 \%$ pentobarbital sodium $(45 \mathrm{mg} / \mathrm{kg})$. The pupils were maximally dilated, and the cornea was topically anesthetized. ${ }^{7,8}$

\section{In vivo method for anti-inflammatory property of curcumin after extraction}

After anaesthesia of rats, Evans blue $(100 \mathrm{mg} / \mathrm{kg})$ was administered by means of rat tail vein. Animals were continued a temperature controlled warming cushion for $2 \mathrm{hr}$, after which they were yielded by $1 \%$ pentobarbital over measurement $>45 \mathrm{mg} / \mathrm{kg}$. The eyes were promptly enucleated and settled with 2\% paraformaldehyde in PBS (Phophate buffer saline) for 2 hrs. Retinas were then analysed, and level sums were mounted on glass slides and envisioned under a confocal magnifying lens.

\section{Selection of Tissue Markers}

Rat Endothelial Cell Antigen Protein (RECA) is important biomarkers in vascular biology. Collagen IV (Col-IV) is an important marker of basement membrane competence in blood vessels and the relationship between Col-IV and diabetes has been extensively studied. ${ }^{27,28}$ Important markers for RGC damage include oxidative stress, Thy-1 (a surface glycoprotein of the immunoglobulin superfamily specifically expressed in RGC), and the transcription factor Brn3a (a transcription factor specifically expressed in cells of the developing mammalian nervous system)..$^{28,29,30,31}$

\section{In vitro method for anti-inflammatory property of Curcumin \\ Western Blotting}

The protein focus in the supernatant was measured and protein (50ug) acquired from each retinal specimen were subjected to SDS-PAGE and electro-phoretially exchanged onto a nitrocellulose membrane. The layer was hindered in 5\% non-fat dried milk arrangement and hatched overnight with incompletely purified rat hostile to VEGF monoclonal antibody, rabbit against ICAM-1 mAb and rabbit antiphospho INOS $\mathrm{mAb}$ against rat ${ }^{16,17,18} \mathrm{CaMKII}$ and NF-kB antibody. Detection of beta actin expression with a mAb was utilized as an inner control to confirm proportionate aggregate protein stacking. ${ }^{4,9,23}$

\section{Immunofluorescence Staining}

After euthanasia, the retina was harvested and immersed in neutral buffered formalin containing $4 \%$ formaldehyde for a period of $6 \mathrm{~h}$, embedded in liquid nitrogen. Sections of $8 \mu \mathrm{m}$ thickness were cut using a freezing microtome. For immunofluorescence, the tissues were cryoprotected in sucrose, frozen and sectioned at $8 \mu \mathrm{m}$ in a cryostat. Slides were incubated successively with blocking solution. The tissue sections were incubated with primary antibody to Rat Endothelial Cell antigen (RECA), Collagen IV antibody (Col-IV), Thy-1 cell surface antigen, Brain-specific homeobox domain protein $3 \mathrm{~A}(\mathrm{Brn} 3 \mathrm{a}),{ }^{29}$ Carbonic Anhydrase II(CA-II). After the hybridization of secondary antibodies, and DAPI staining for the cell nucleus, the sections were observed at the fluorescence microscope (LeCurcumin DM 6000 Laser Station). Semiquantitative analysis was performed to evaluate the intensity of RECA, Col-IV, Thy-1, Brn3a, and CA-II staining using Image pro plus software, ${ }^{22,25}$

\section{Retina Ganglion Cell Tissue Culture In vitro}

The retinal ganglion tissue of sham group and DM placebo group (untreated) $(n=9)$ were cultured in vitro, stimulation with different concentration of curcumin and the length of neurite outgrowth was measured. Each retinal tissue specimen was divided into two sections. After PBS rinsing, the freshly dissected retina ganglion fragment was placed on a coverslip, to which a $14 \mu \mathrm{L}$ drop of growth-factor-reduced Matrigel $^{\mathrm{Tm}}$ had been added and kept in liquid formusing a cold $35-\mathrm{mm}$ plastic culture dish on ice. The growth factor reduced Matrigel ${ }^{\mathrm{Tn}}$ was polymerized $\left(5-\mathrm{min}\right.$ incubation at $37^{\circ} \mathrm{C}$ ) and $2 \mathrm{~mL}$ of serum-free RPMI1640 added. Tissue fragments from the control and diabetic group were treated at various concentrations of curcumin for 3 days. Ganglial cultures were maintained at $37^{\circ} \mathrm{C}$ in a humidified atmosphere with $5 \%$ $\mathrm{CO} 2$. Photographs of neurite growth at 6 days were captured using a Nikon DXM 1200 digital still camera attached to LeCurcumin Laborlux microscope and ACT-1 software. Digital images were analysed using Image-Pro Plus software to determine the longest neurite length per specimen. Mean maximal neurite length was calculated for the control and diabetic group by averaging the longest neurite length from each individual specimen.

\section{Statistical Analysis}

Results were expressed as means \pm standard deviation. One-way ANOVA followed by Bonferron multiple comparisons' test was used to evaluate whether differences between groups were significant. All calculations were performed using SPSS statistical software. Probability values of less than $5 \%$ were considered significant.

\section{RESULTS}

\section{Curcumin changes morphology of retina in DM rats}

Comparing placebo treated diabetic to control animals, numerous Morphological changes were observed in inner nuclear layer (INL), outer nuclear layer (ONL), retinal ganglion cells (RGCs), and the intensity and number of bipolar cells in the INL and ONL. RGC were considerably reduced in diabetic group as compared with those of the controls. The thickness of the basal membrane in diabetic group was significantly decreased $(76.18 \pm 5.2$ um vs. $67.12 \pm 4.8$ um). In the Curcumin group, the thickness of basal membrane was $73.53 \pm 4.1 \mathrm{um}(P<0.05)$. The morphological structure of the retinal specimens was qualitatively better in the curcumin treated group (Figure 4) 


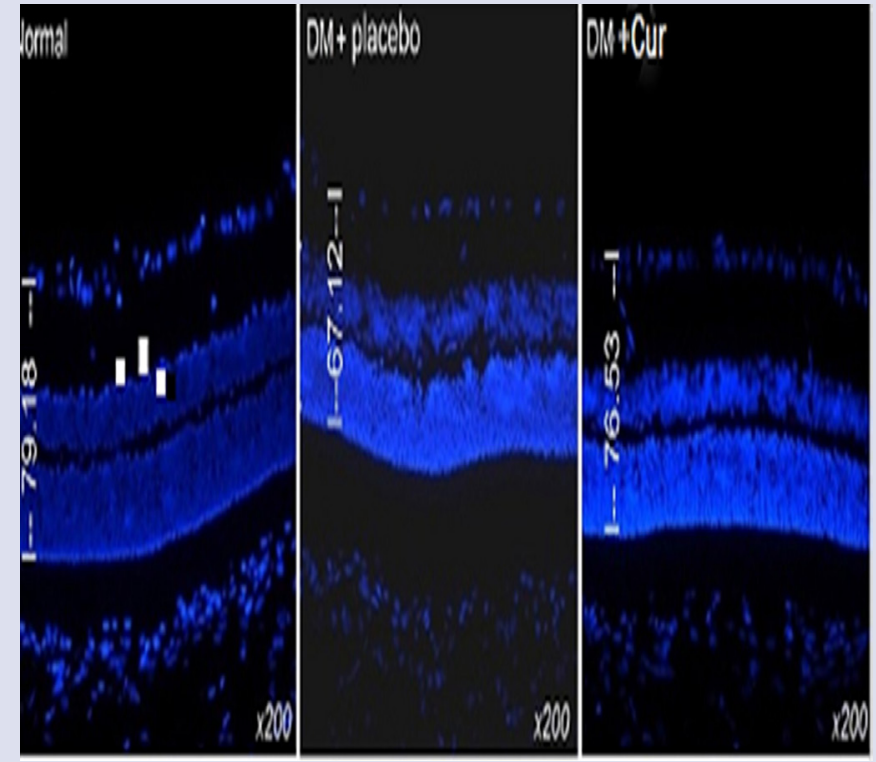

Figure 4: The effect of curcumin on morphological changes of diabetic retinopathy.The notable morphological changes were observed in diabetic retina, however, these changes were improved by curcumin treated diabetic retina and the average thickness of retina in different groups.

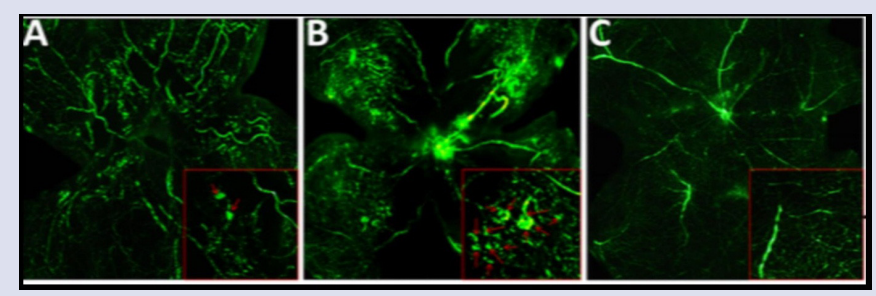

Figure 5: (Evans blue fluorescence. (1) Representative image of retinal fluorescence in an untreated control animal (Con), in which Evans blue fluorescence is limited to the blood vessels. (2) In Alloxan-treated diabetic retinas (DM), dye leakage is observed (white arrows). This leakage was not seen in Alloxan-treated diabetic rats administered (3) curcumin(DM+Cur).

\section{Curcumin inhibits retinal vascular leakage induced by diabetes}

Evans blue was utilized as a part of retinal level adds up to assess the impact of curcumin on retinal vein leakage. In control retinas, Evans blue fluorescence was situated inside blood vessel (Figure 5.1). But in alloxan treated rats (Figure 5.2) leakage of colour from vessels and bigger vessels were noted. This spillage was likewise not seen in alloxan treated rats which were controlled by curcumin (Figure 5.3). Evans blue level were lifted in the retinas of alloxan regarded diabetic rats when contrasted with control rats with decreased vascular leakage. This rise was essentially diminished in alloxan treated rats with administration of curcumin. ${ }^{12}$

\section{Curcumin improve blood vessels density}

Retinal blood vessels are clearly defined in retinal pigment epithelium (Figure 6). Collagen IV expression was less in the diabetic retina, consistent with thickening of the micro-vessel basement membrane. Basement membrane thickening was less in the curcumin treated
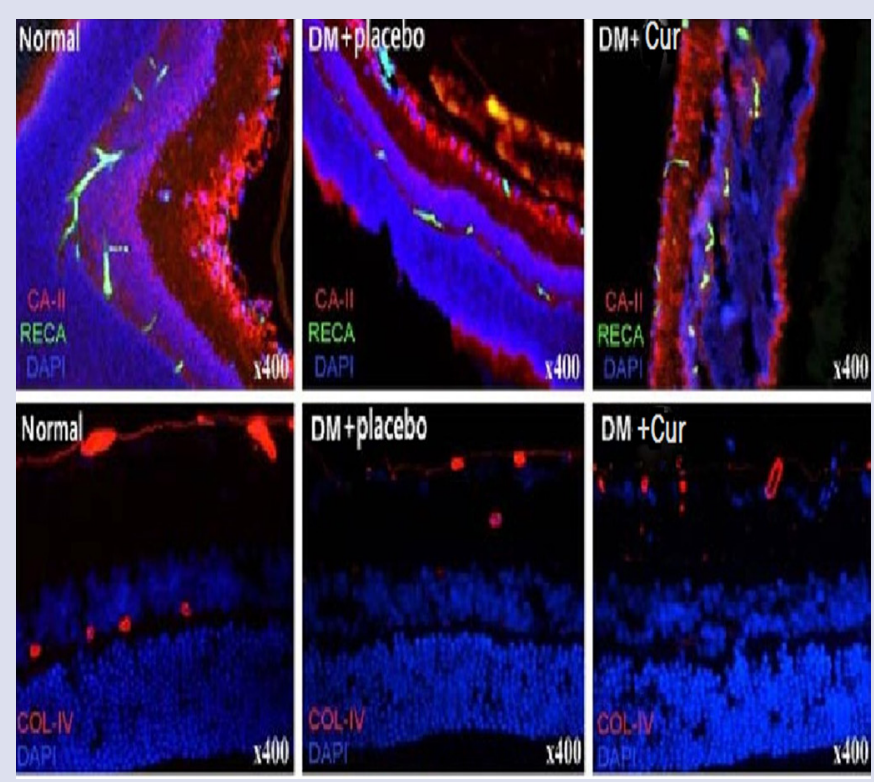

Figure 6 (a): In diabetic retina RECA,Col-IV and VEGF were used to check the retinal microvessels. RECA and Col-IV clearly demonstrated the distribution of retinal vessels.DM decreased the blood vessels density in retina while curcumin improved these conditions. Curcumin also improved the expression of VEGF significantly.

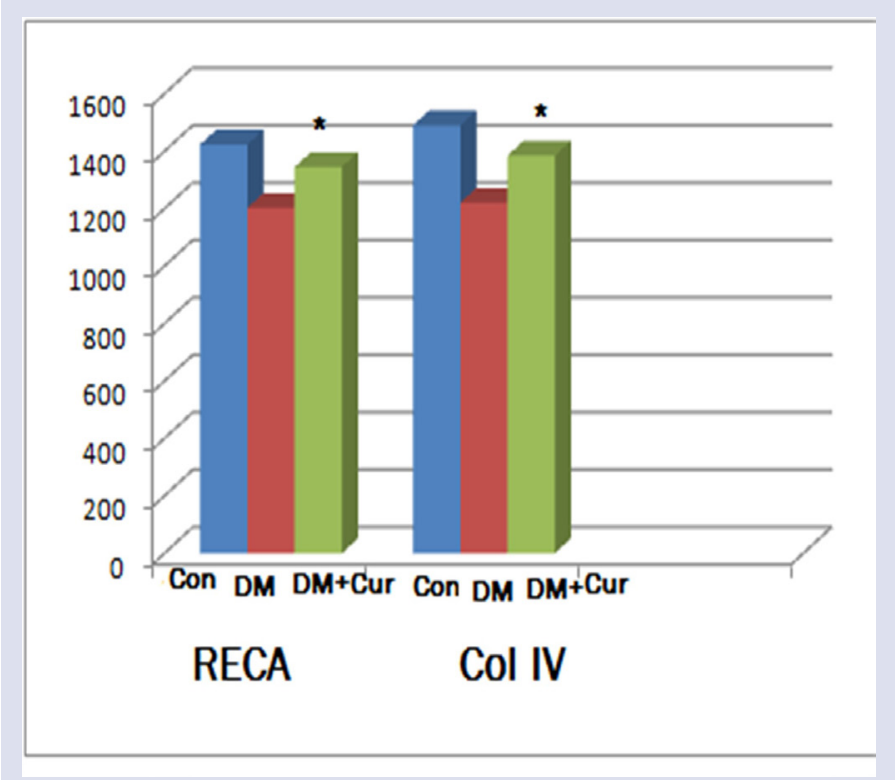

Figure 6 (b): Graphical representation of RECA and Col IV.

diabetic group compared to placebo-treated diabetic animals. Curcumin treated animals also had greater expression of RECA and microvessel density (Table 1, Figure 6a and b)

\section{Curcumin increases Retinal Ganglionic Cells in DM rats}

Thy-1 and Brn3a expression in diabetic retinas were significantly decreased in the inner nuclear layer, outer nuclear layer ONL), retinal ganglion cells compared to sham control retinas during oxidative stress condition inside the body. Diabetic rats treated with curcumin had greater expression of Thy-1 and Brn3 relative to placebo treated diabetic 
rats. Muller cells support neuronal activity and integrity of blood retinal barrier, whereas alternation of muller cells under pathological condition may contribute to retinal degeneration and oedema formation. CA-II was used to detect muller cells in rats treated with curcumin. ${ }^{26}$ (Table 2, Figure $7 \mathrm{a}$ and $\mathrm{b}$ )

Table 1: Effects of curcumin on Col IV and RECA.

\begin{tabular}{ccc}
\hline Groups & RECA & Col IV \\
\hline Normal & $1423 \pm 85$ & $1490 \pm 123$ \\
DM+Plaebo & $1202 \pm 97$ & $1220 \pm 190$ \\
DM+Cur & $1345 \pm 84^{*}$ & $1485 \pm 105^{\star}$ \\
P value & $<0.05$ & $<0.05$ \\
\hline
\end{tabular}

RECA, Col IV were used to used check retinal micro vessels. Values are the mean values from $\mathrm{N}=3$ animals per group. ${ }^{\star} \mathrm{P}<0.05,{ }^{* *} \mathrm{P}<0.01$ compared with the placebo group.

Table 2: The effects of Curcumin on RGCs Thy-1 and Brna-3 and CA II expression in Diabetic retina.

\begin{tabular}{cccc}
\hline Groups & CA-II & Thy-1 & Brna3 \\
\hline Normal & $1720 \pm 101$ & $2090 \pm 143$ & $1923 \pm 98$ \\
DM+Placebo & $1490 \pm 192$ & $1756 \pm 87$ & $1365 \pm 65$ \\
DM+Cur & $1560 \pm 121^{*}$ & $1870 \pm 110^{* *}$ & $1509 \pm 126^{* *}$ \\
P value & $<0.05$ & $<0.01$ & $0<01$ \\
\hline
\end{tabular}

Thy-1 and Brn3a were used to detect the RGC, CA-II was used to detect the mullercells. Values are the mean values from N-9 animal group. ${ }^{*}<0.05,{ }^{* *} 0.01$ compared with the placebo group.

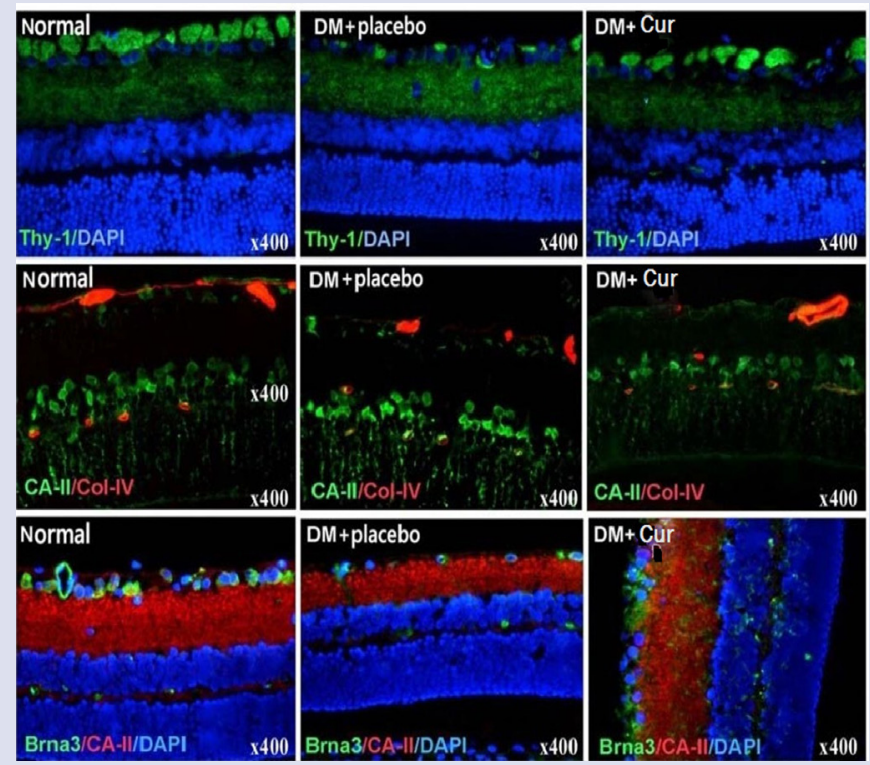

Figure 7 (a): Thy-1 and Brn3a expression in diabetic retinas were significantly decreased in the inner nuclear layer,outer nuclear layer $\mathrm{ONL}$ ), retinal ganglion cells) compared to sham control retinas during oxidative stress condition Diabetic rats treated with curcumin had greater expression of Thy- 1 and Brn3 relative to placebo treated diabetic rats. CA-II was used to detect muller cells in rats treated with curcumin.

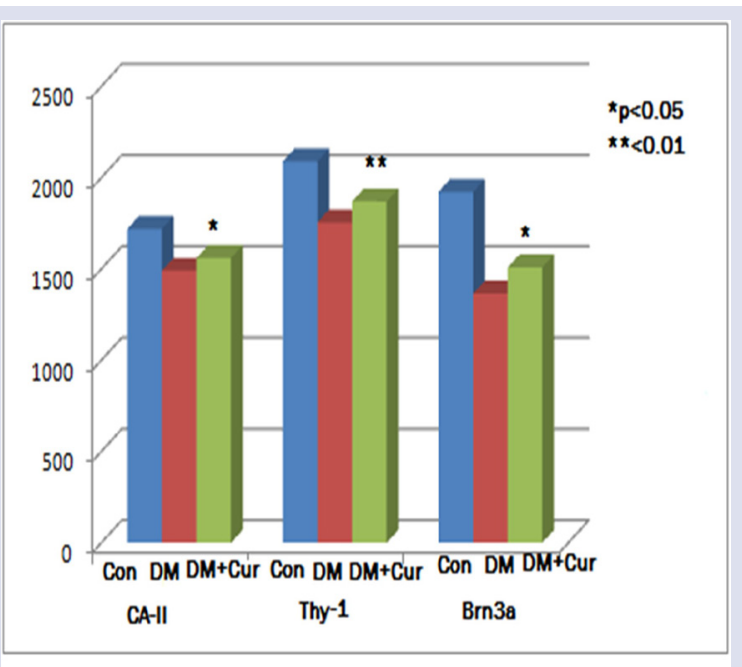

Figure 7 (b): Graphical representation of CA II, Thy-1, Brn3a expressions.

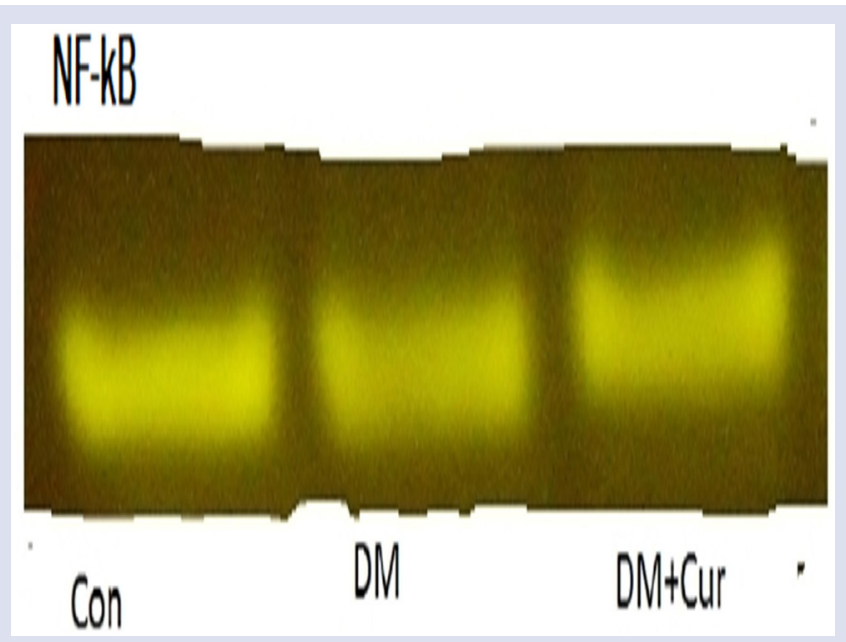

Figure 8 (a): Western blot analysis of retinal levels of p65 subinit of NF-kB obtained for control rats (Con), and alloxan-treated diabetic rats administered vehicle (DM), curcumin (DM+Cur).

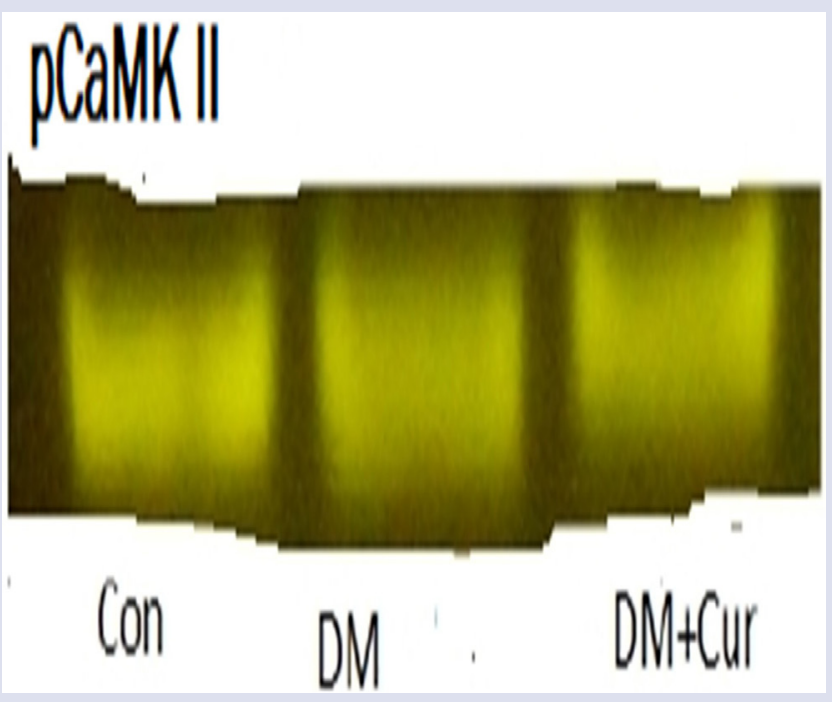

Figure 8 (b): Western blot analysis of retinal levels of p CaMK II obtained for control rats (Con), and alloxan-treated diabetic rats administered vehicle (DM), curcu $\neg \min (\mathrm{DM}+\mathrm{Cur})$. 
Table 3: Western blot quantification of p-65 subunit of NF-kB and CaMKII.

\begin{tabular}{ccccccc}
\hline \multicolumn{7}{c}{ Protein expression of p65 subunit NF-kB and CaMK II } \\
\hline & \multicolumn{1}{c}{$1^{\text {st }}$ group of rats } & \multicolumn{2}{c}{$\mathbf{2}^{\text {nd }}$ group rats } & \multicolumn{2}{c}{$\mathbf{3}^{\text {rd }}$ group of rats } \\
\hline NF-kB & CaMK II & NF-kB & CaMKII & NF-kB & CaMKII \\
\hline Con & 0.7 & 1.3 & 0.8 & 1.4 & 0.6 & 1.2 \\
DM & 1.3 & 2 & 1.2 & 3 & 1.4 & 1 \\
DM+Cur & 0.9 & 1.3 & 1 & 1.4 & 0.8 & 1.2 \\
\hline
\end{tabular}

Table 4: Quantification of signal intensity of VEGF, iNOS and ICAM-1 proteins of retinal tissue.

\begin{tabular}{|c|c|c|c|c|c|c|c|c|c|}
\hline \multicolumn{10}{|c|}{ Retinal Cytokine levels } \\
\hline & \multicolumn{3}{|c|}{ Ist group rats } & \multicolumn{3}{|c|}{$2^{\text {nd }}$ group } & \multicolumn{3}{|c|}{$3^{\text {rd }}$ group } \\
\hline & VEGF & iNOS & ICAM-1 & VEGF & iNOS & ICAM-1 & VEGF & INOS & ICAM-1 \\
\hline $\mathrm{cON}$ & 0.8 & 1.2 & 1.5 & 1 & 1.3 & 1.5 & 0.6 & 1.3 & 1.7 \\
\hline DM & 1.5 & 2 & 1.6 & 1.6 & 1.2 & 1.4 & 1.4 & 2.8 & 1.8 \\
\hline $\mathrm{DM}+\mathrm{Cur}$ & 1.2 & 1.3 & 1.6 & 1.4 & 1.1 & 1.2 & 1 & 1.6 & 1.7 \\
\hline
\end{tabular}

Western blot analysis of retinal levels of NF-kB \& . CaMKII

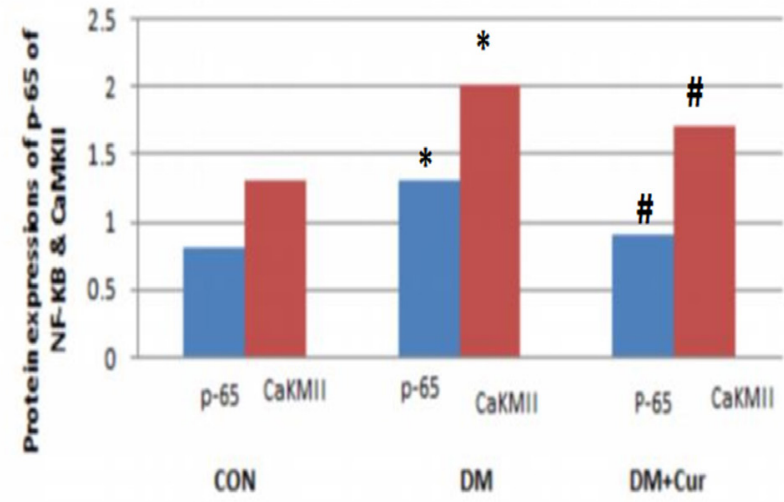

Figure: 8 (c)Graphical representation of Western blot quantification of $\mathrm{p}-65$ subunit of NF-kB and CaMK II Indicate average +SEM from atleast three independent experiments ${ }^{*} \mathrm{p}<0.05$ versus con; $\# \mathrm{p}<0.05$ versus $D M$.

\section{Curcumin suppreses phosphorylation NF-kB p65 through CaMKII pathway in diabetic retina}

Phosphorylation of p65 subunit of NF-kB plays an important role regulating expression and encode pro-inflammatory cytokines and adhesion molecules through activation of CaMKII dependent pathway in diabetic retina. ${ }^{12}$ But administration of curcumin $(100 \mathrm{mg} / \mathrm{kg} /$ day $)$ decreased phosphorylation p65subunit of NF-kB by inhibiting enzyme CaMKII activity (Figure $8 \mathrm{a}$ and $\mathrm{b}$ ) which were significantly elevated in retinas of Alloxan treated diabetic rats as compared to controls. ${ }^{13}$

\section{Curcumin reduce VEGF, iNOS and ICAM-1 expressions in} the diabetic retina

After decreasing expression of encoder NF-kB for pro inflammatory cytokines, effect of curcumin on the expression levels of VEGF, iNOS, ICAM-1cytokines were measured. These inflammatory cytokines

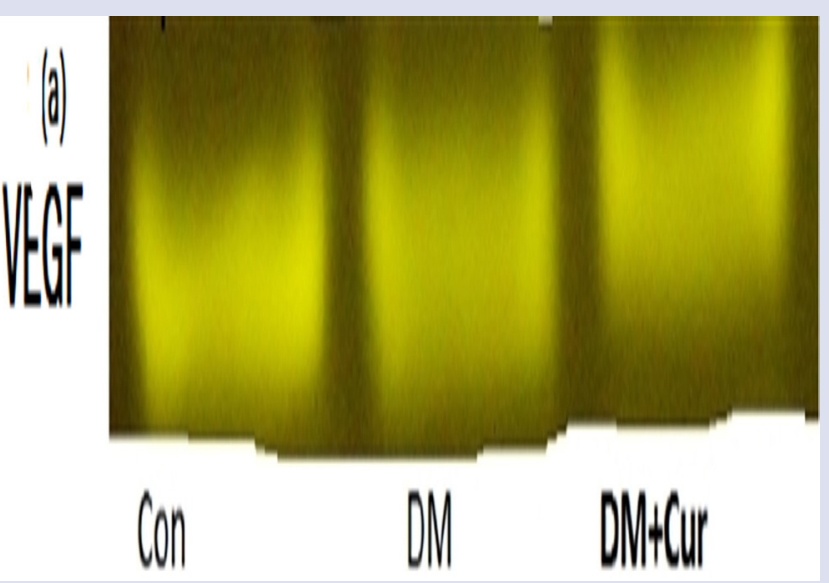

Figure 9 (a): FRetinal cytokine levels for VEGF,INOS,ICAM-1 in nondiabetic control rats (Con), alloxan-treated dia $\neg$ betic rats administered vehicle (DM), curcumin (DM+Cur) . Results are expressed relative to average values measured in control re $\neg$ tinas. Representative western blots for VEGF protein expression in the 3 experimental groups.

expression were signiffcantly reduced by administration of curcumin as shown Figure $9 \mathrm{a}$ and $\mathrm{b}^{24,30}$

\section{Effects of Curcumin on RGC Neurite Outgrowth from Retina in Vitro during oxdative stress condition}

Neurite outgrowth was measured in cultured RGC from diabetic and normal rats. Paired comparisons were made between retina derived from normal control and DM rats at the treatment of $0,10,100$ and $1000 \mathrm{nmol} / \mathrm{mL}$ crucumin at the $72 \mathrm{~h}$ time point, all retina treated with curcumin had signif Curcuminntly longer average neurite length when compared to DM group. (Table 5, Figure $10 \mathrm{a}$ and b)

\section{DISCUSSION}

This is the main report demonstrating that curcumin, a polyphenol has gainful impact on retinal metabolic anomalies including oxidative stress 


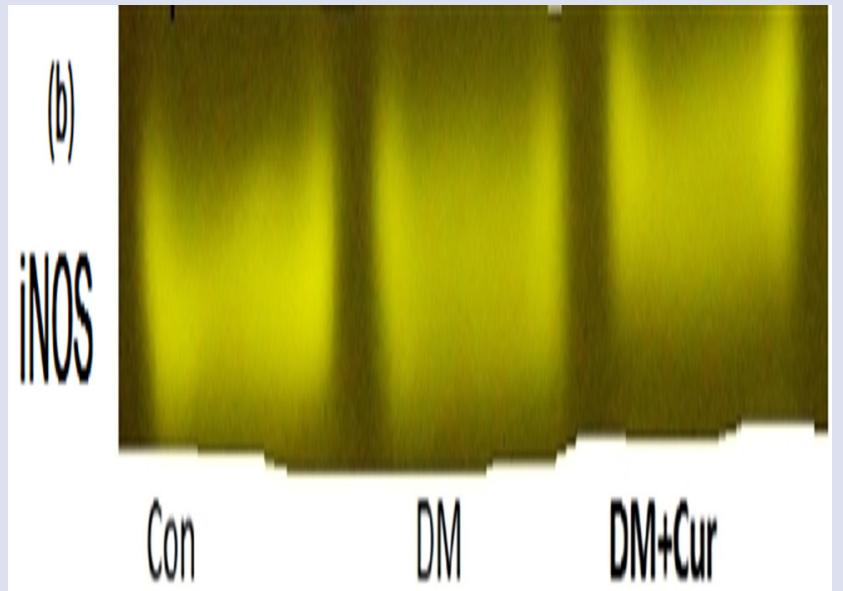

Figure 9 (b): Retinal cytokine levels for VEGF,INOS,ICAM-1 in nondiabetic control rats (Con), alloxan-treated dia $\neg$ betic rats administered vehicle (DM), curcumin (DM+Cur). Results are expressed relative to average values measured in control re $\neg$ tinas. Representative wes $\neg$ tern blots for iNOS protein expression in the 3 experimental groups.

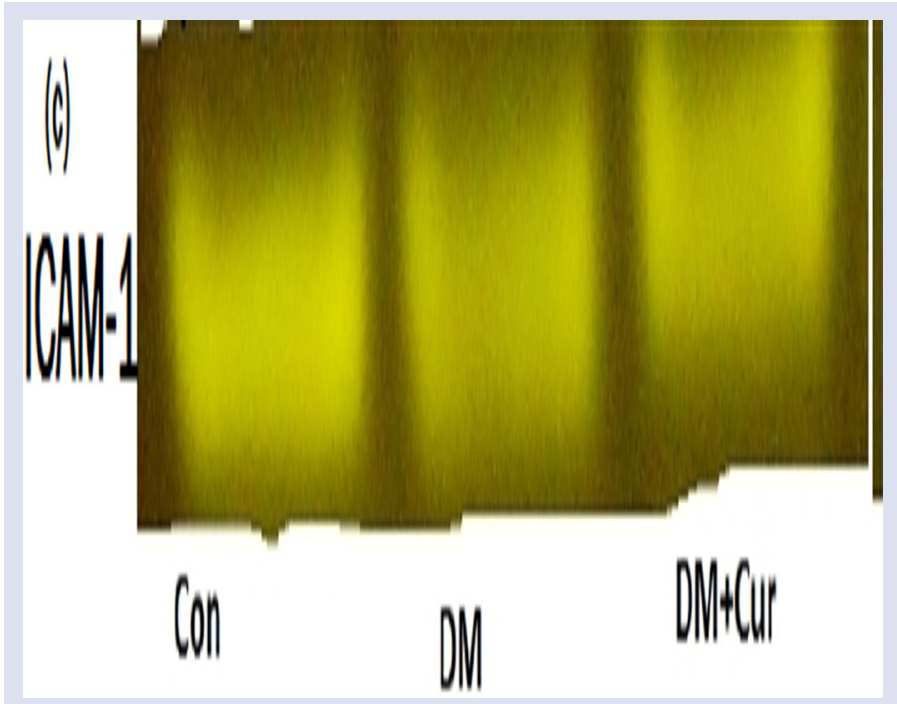

Figure $\mathbf{9}$ (c):Retinal cytokine levels for VEGF,INOS,ICAM-1 in nondiabetic control rats (Con), alloxan-treated dia $\neg$ betic rats administered vehicle (DM), curcumin (DM+Cur) . Results are expressed relative to average values measured in control re $\neg$ tinas. Representative wes $\neg$ tern blots for ICAM-1 protein expression in the 3 experimental groups.

and inflammation, which is thought to be essential in the improvement of retinopathy in diabetes. We provided the proof that administration of curcumin with vasoactive properties to a rodent model of diabetes can successfully stifle the activity of transcription factor NF-kB through restraint of CaMKII. We likewise demonstrated that this activity downregulates the incendiary cytokines VEGF, INOS and ICAM-1. These changes are joined by diminished vascular penetrability of veins in the diabetic retina. In these studies, we additionally found that curcumin eases the blood retinal barrier(BRB) spillage observed in alloxan treated rats. Inhibition of vascular injuries by curcumin was independent of progress in blood glucose level. In the present study, we also provided evidence that curcumin may be of benefit in the Diabetic

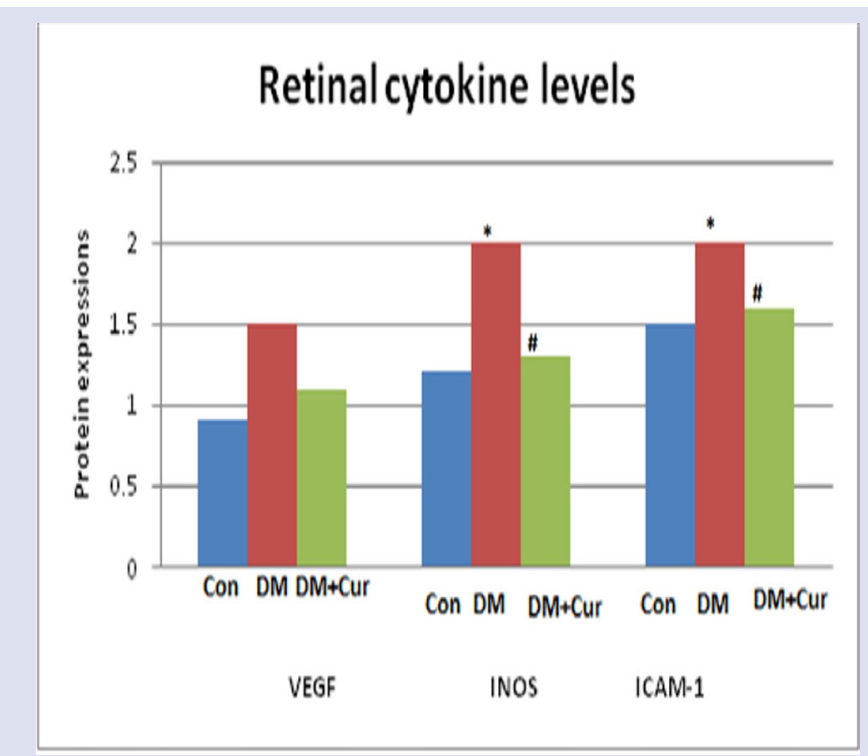

Figure $\mathbf{9}$ (d): Graphical representation of quantification of signal intensity ofVEGF,iNOS and ICAM-1 proteins of retinal tissue indicate average + SEM of at least 3 retinas per group. ${ }^{*} p<0.01$ versus Con; $\# p<0.01$ versus DM.

Table 5: The neurites sprout from retina after stimulation with curcumin in normal rats and diabetic rats.

\begin{tabular}{ccccc}
\hline Group & Sham & $10 \mathrm{nM}$ & $100 \mathrm{nM}$ & $1000 \mathrm{nM}$ \\
\hline Normal & $170 \pm 3.5^{* *}$ & $255 \pm 1.5^{* *}$ & $272 \pm 2.4^{* *}$ & $311 \pm 3.4^{* *}$ \\
DM & $102 \pm 4.8$ & $129 \pm 2.3$ & $154 \pm 4.1$ & $178 \pm 3.8$ \\
P Value & $<0.01$ & $<0.01$ & $<0.01$ & $<0.01$ \\
\hline
\end{tabular}

Paired comparisons were made between retina derived from normal control and DM rats at thetreatment of $0,10,100$ and $1000 \mathrm{nmol} / \mathrm{mL}$ CURCUMIN at the $48 \mathrm{~h}$ time point. Values are the mean values ( \pm standard deviation) from $N=12$ animals per group. ${ }^{*} P<0.01$ compared with the DM group.

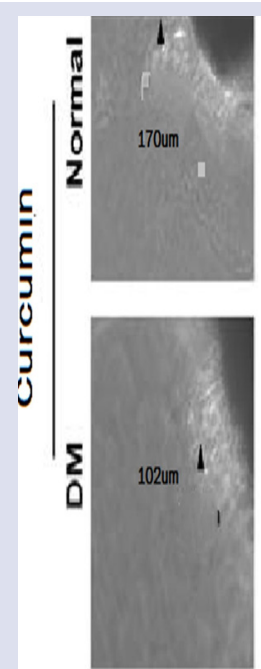

Sham
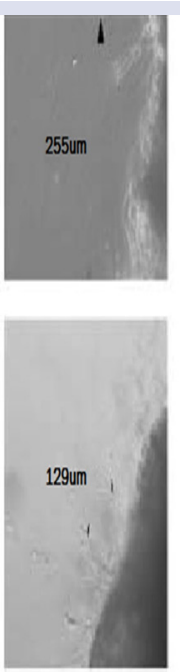

$10 \mathrm{nmol} / \mathrm{ml}$
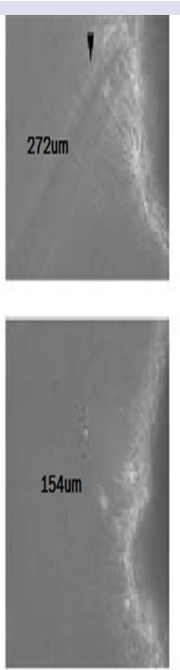

$100 \mathrm{mmol} / \mathrm{ml}$
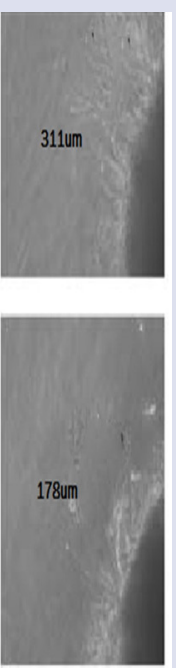

$1000 \mathrm{nmol} / \mathrm{ml}$
Figure 10 (a): Neurite outgrowth was measured in cultured RGC from diabetic and normal rats. Paired comparisons were made between retina derived from normal control and DM rats at the treatment of 0 , 10,100 and $1000 \mathrm{nmol} / \mathrm{mL}$ crucumin at the $72 \mathrm{~h}$ time point, all retina treated with curcumin had significantly longer average neurite length when compared to DM group. 


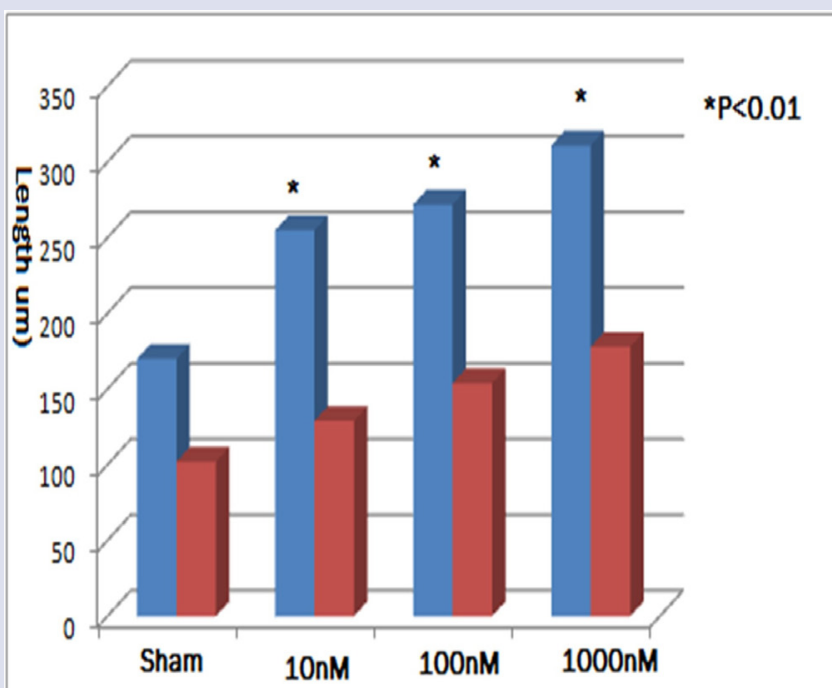

Figure 10 (b): The neurites sprout from retina after stimulation with Curcumin in normal rats and diabetic rats. Paired comparisons were made between retina derived from normal controland DM rats at the treatment of $0,10,100$ and $1000 \mathrm{nmol} / \mathrm{mL}$ Curcumin, all retina treated with curcumin had significantly longer average neurite length in different dose of curcumin in normal rats and diabetic rats when compared to DM group.

retinopathy prevention in diabetes. Curcumin treated diabetic rats had significantly greater microvessel density, thinner basement membranes, greater expression of endothelial and ganglia markers, compared to the control group. Importantly, there was no significant difference in metabolic and physiological variables between the diabetic control and curcumin treated group, implying that these effects showed independent of serum glucose level.

Retinal micro vascular changes in Diabetic retinopathy might be related to hyperglycemia-induced intra mural pericyte death and thickening of the basement membrane. This may lead to incompetence of the vascular wall sand disruption of the blood-retinal barrier. ${ }^{18}$ In our study, retinal microvessel density is significantly decreased in diabetic animals compared to normal. Endothelial deterioration and neuropathy are also important pathological process in diabetic retinopathy models. Intracellular hyperglycemia has been linked to an overproduction of ECM; hyperglycemia may also decrease production of trophic factors for endothelial and neuronal cells, and the intracellular oxidative stress in endothelial cells plays a key role in endothelial dysfunction. ${ }^{8,9,15,19,20,32}$ Together, these changes lead to neuropathy and endothelial lesions. Collagen IV is one of the major components in the blood vessel basement membrane. The expression of collagen IV during diabetes has been extensively studied. It has been reported that collagen IV is highly expressed in the diabetes and related to many diabetic pathological disorders. ${ }^{27}$ The major expression of RECA is related with their vascularization and many clinical studies have shown a strong correlation between increases in intraocular RECA concentration and the development of proliferative diabetic retinopathy. Interestingly, our results demonstrate that Col IV, RECA expression in the retina were decreased in diabetic rats compared to normal controls. Cultured retinal ganglion is an important and almost an indispensable tool for the study of retinal visual physiology and pathophysiology, and it may easily evolve as state-of-art technology for studying the inter-cellular or intra-cellular processes associated with various retinopathies and neuropathies. ${ }^{27,28}$

In our study curcumin appeared to enhance neurite length in both normal and diabetic rat retinas. This effect was dose dependent, with greater concentrations leading to greater mean neurite length.

\section{CONCLUSION}

CaMKII reacts to increment in $\mathrm{Ca} 2+$, resulting from incitement of NMDA receptors. The elevation of VEGF expression and BRB breakdown in alloxan prompted diabetic rats is hindered by NMDA receptor antagonist. ${ }^{17}$ Our work has demonstrated that curcumin can lift Retinal Ganglionic Cells (RGC) substance to near normal levels in diabetic rats by modulating Thy-1 and Brn3a expression. Curcumin may also be useful in the management of DR by modulating both RECA and Col-IV expression in retinal microvessel. These perceptions show that high glucose initiates diminish retinal ganglionic cells that thus enact CaMKII a NF-kB, Brn3a and consequently raise cytokine expression and ensuing retinal vascular spillage in diabetes. This model is bolstered by our perceptions that high glucose-actuated articulation of cytokines (INOS, ICAM-1 and VEGF) was blocked by both NF-kB and CaMKII inhibitor i.e Curcumin. ${ }^{17,18,19,20}$

\section{REFERENCES}

1. Adamis AP. Is diabetic retinopathy an inflammatory disease? $\mathrm{Br} \mathrm{J}$ Ophthalmol. 2002;86(3):363-5.

2. Bloomgarden ZT. Screening for and managing diabetic retinopathy: current approaches. Am J Health Syst Pharm. 2007;64:S8-14.

3. Pradhan D, Pradhan RK. Investigation of in-vivo anti-oxidant property of S Cumini. In streptozocin induced diabetic rats. Journal pharmaceut Curcuminl science and pharmacology. 2015;2:134-9.

4. PradhanD, Tripathy G, Pradhan S, Dasmohapatra T. A review of Cuminoside nano medicine-Pharma cognostic approach to cancer therapeutics. Journal Young Pharmacist. 2016;8(2):61-7.

5. Kern TS, Contributions of inflammatory processes to the development of early stages of diabetic retinopathy. 2007;95-103.

6. Kowluru RA, Kern TS. Abnormalities of retinal metabolism in diabetes or experimental galactosemia IV. Antioxidant defence system. Free Rad Bial Med. 1997;22(4):587-92.

7. Patanaik S, Pradhan D, Tripathy G. Ant Curcuminncer activity Limoniaacidissima, cancer cellines. 2012;3(3):413-9

8. SKS, Tripathy G, Pradhan D. Anti-diabetic and anti-oxidant of Spindus Trifoliatus linn. 2010;1(2):1-7.

9. Thrope SR. Role of oxidative stress in diabetic compl Curcumintions. A new perspective on an old paradigm. 1999;48(1):1-9.

10. SAHOO PK, Pradhan D, Behera P. Pharmacology Curcuminl invention. Adenosine receptor in Diabetic neuropathic pain. 2010;3(8):1798-802.

11. Pradhan S, Pradhan D, Tripathy G. Ethnopharmacology Curcuminl approach in exctraction of bioactive compound. $2015 ; 7(1): 42-9$

12. Yousif $\mathrm{MH}$, Benter IF, Walther T. Role of ca2+/calmodulin-dependent protein kinase II normalises diabetes induced abnormal vascular reactivity in the rat perfused mesenteric vascular bed: Auton Autacoid Pharmacol. 2003;23(1):27-33.

13. Anjana M, Pradhan D, Tripathy S. Anti-inflammatory and anti-arthritic potential of Amaniabacifera linn. IJPBS. 2010;1(3):1-7.

14. Dasmohapatra T, Pradhan D, Tripathy G. Pharmacognostic evaluation of DPSUU III in breast tumor growth by G0/G1 Cell cycle arrest. 2017;51(1):70-6.

15. Zhang SX, Ma JX, Sima J, Hu MS. Genetic difference in susceptibility to the blood retinal barrier breakdown in diabetes and oxygen induced retinopathy. Am j pathol. 2005; 166(1):313-21.

16. Pradhan D, Panda PK, Tripathy G. Ant Curcuminncer activity of Biflavanoids Cancer cell lines. Journal pharmacy research. 2009;2(5):40-5.

17. Zheng L, Du Y, Miller C, Kern TS. Crit Curcuminl role of inducible nitric oxide synthatase in degeneration of retinal capillaries in mice with streptozocine induced diabetes. Diabetologia. 2007;50:1987-96.

18. Wiess $A$, Brownlee $M$ et al. Secondary intervention with amino guanidine retards the progression of diabetic retinopathy in the rat model. Diabetologia. 1995;38(6):656-60.

19. Inhibition of mammary cell carcinoma reverse multi drug resistance by SOCSI Pharmacognosy journal. 2014;8(2).

20. Wallace TM, Cohen $C$, et al. Macrophage and retinal pigment epithelium expression of angiogenic cytokines in choroidal neovascularizationn. Mol Vis. 
2002;8(8):119-26.

21. Thompson KH, Bohmerle K, Polishchuk E, Martins C, Toleikis P, Tse J, et al. Complementary inhibition of synoviocyte, Smooth muscle cell or mouselymphoma cell proliferation by a vanadyl curcumin complex compared to curcumin alone. J Inorg. Biochem. 2004;98(12):2063-70.

22. Marshall SM, Flyvbjerg A. Prevention and early detection of vascular compl Curc umintions of diabetes. Br. Med. J. 2006;333:475-80.

23. Antonetti DA, Barber J, Bronson SK, La Noue KF, et al. JDRF Diabetic Retinopathy Centre Group. Diabetic retinopathy: Seeing beyond glucose-induced microvascular disease. Diabetes. 2006;55(9):2401-11.

24. Liu WJ, Xin ZC, Xin H, Yuan YM, Tian L, Guo YL. Effects of Curcuminriin on erectile function and expression of nitric oxide synthase isoforms in castrated rats. Asian J. Androl. 2005;7(4):381-8.

25. Shindel AW, Xin ZC, Lin G, Fandel TM, Huang YC, Banie L. Erectogenic and neurotrophic effects of Curcuminriin, a purified extract of horny goat weed (Epimedium spp.) in vitro and in vivo. J. Sex. Med. 2010;7(4pt1):1518-28.

26. Reichenbach A, Wurm A, Pannicke $T$, landiev I, Wiedemann P, Bringmann A. Muller cellsas players in retinal degeneration and edema. Graefes Arch. Clin. Exp. Ophthalmol. 2007;245(5):627-36.

27. Li Q, Ao X, DuY, Li Y, Ou Y, Gong R. Effects of aminoguanidine and vitamin C on collagen type IV in diabetic nephropathy rats. Endocrine. 2011;39(3):251-8.

28. Sivakumar V, Zhang Y, Ling EA, Foulds WS, Kaur C. Insulin-like growth factors, angiopoietin-2, and pigment epithelium-derived growth factor in the hypoxic retina. J Neurosci. Res. 2008;86(3):702-11.

29. Sanchez-Migallon MC, Nadal-Nicolas FM, Jimenez-Lopez M, Sobrado-Calvo P,Vidal-Sanz M, Agudo-Barriuso M. Brain derived neurotrophic factor maintains
Brn3a expression in axotomized rat retinal ganglion cells. Exp. Eye Res. 2011; 92(4):260-7.

30. Li J, Wang JJ, Yu Q, Chen K, Mahadev K, Zhang SX. Inhibition of reactive oxygen species by Lovastatin downregulates vascular endothelial growth factor expression and ameliorates blood-retinal barrier breakdown in $\mathrm{db} / \mathrm{db}$ mice: Role of NADPH oxidase 4. Diabetes. 2010;59(6):1528-38.

31. Shahidi AM, Sampson GP, Pritchard N, Edwards K, Russell A, Malik RA, et al, Exploring retinal and functional markers of diabetic neuropathy. Clin. Exp. Optom. 2010;93(5):309-23.

32. Zhang W, Liu H, Al-Shabrawey M, Caldwell RW, Caldwell RB. Inflammation and diabetic retinal microvascular complCurcumintions. J. Cardiovasc. Dis. Res. 2011;2(2):96-103

33. Klein R, Klein BE, Moss SE. Epidemiology of proliferative diabetic retinopathy DiabetesCare. 1992;15(12):1875-91.

34. Frank RN. Diabetic retinopathy. N. Engl. J. Med. 2004;350:48-58.S

35. Curtis TM, Gardiner TA, Stitt AW. Microvascular lesions of diabetic retinopathy: Clues toward Sunderstanding pathogenesis? Eye (Lond). 2009;23(7):1496-508.

36. Pambianco G, Costacou T, Ellis D, Becker DJ, Klein R, Orchard TJ. The 30-year natural history of type 1 diabetes compl Curcumintions. The Pittsburgh epidemiology of diabetes compl Curcumintions study experience. Diabetes. 2006;55(5):1463-9.

37. Wright T, Cortese F, Nilsson J, Westall C. Analysis of multifocal electroretinograms from a population with type 1 diabetes using partial least squares reveals spatial and temporal distribution of changes to retinal function. Doc Ophthalmol. 2012;125(1):31-42

\section{GRAPHICAL ABSTRACT}

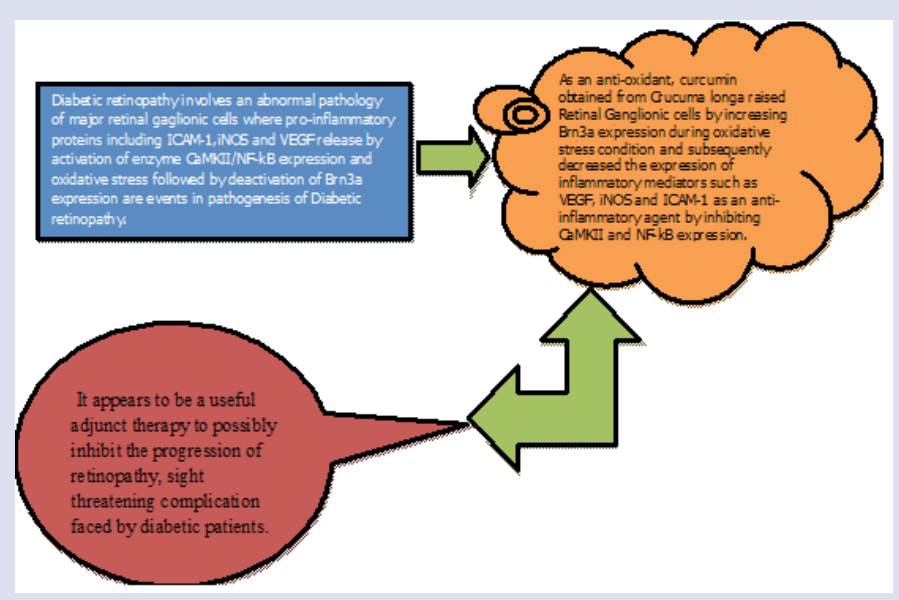

\section{SUMMARY}

- Diabetic retinopathy is microvascular complication of diabetes and involves an abnormal pathology of retinal ganglionic cells where activation of enzyme CaMKII and NF-kB expression occur.

- Diabetic induced oxidative stress followed by deactivation of Brn3a expression in the retinal ganglionic cells are also pathogenesis of Diabetic retinopathy.

- As an anti-oxidant, curcumin raised Retinal Ganglionic cells by increasing Brn3a expression and by inhibiting CaMKII / NF-kB expression.

- $\quad$ Curcumin can be used as a therapy against diabetic retinopathy. against diabetic retinopathy.

\section{ABOUT AUTHORS}

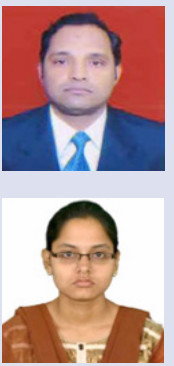

Dr.DebasishPradhan: Is Specialist in Immunity and Breast Cancer Medicine. He is currently working in Department of pharmaceutical sciences,Creighton University, 2500 Carlifornia Plaza,Omaha,NE68102,United States $\mathrm{He}$ is a recognized expert in nutritional medicine, immunity and cancer. Nine no of PhD degree have been awarded under his Supervision. He has written over 75 articles on natural medicines and one book "Fundamentals and principles in Pharmacology". He has all the degrees and publication in the research of EthnoMedicine/ Herbal Medicine/ Natural Medicine and a total experience of thirteen years.

Miss.Toffa Dasmohapatra: is a bonafied student of Department of Pharmaceutical Sciences, UtkalUniversity. She is continuing as a PG student and continuing research as a co-researcher.

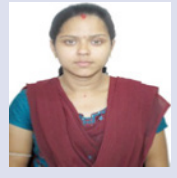

Dr. Gitanjali Tripathy: Is serving as a lecturer at Govt Polytechnic, Pharmacy Dept., Bhubaneswar. She has written two books and 30 publication to her credits. She is a co researcher in this article.

Cite this article: Pradhan D, Dasmohapatra T, Tripathy G. Pharmacognostic Evaluation of Curcumin on Diabetic Retinopathy in Alloxan-induced Diabetes through NF-KB and Brn3a Related Mechanism. Pharmacog J. 2018;10(2):324-32. 